\title{
Subthreshold Periodic Signal Detection by Bounded Noise-Induced Resonance in the FitzHugh-Nagumo Neuron
}

\author{
Yuangen Yao, ${ }^{1,2}$ Lijian Yang, ${ }^{3}$ Canjun Wang, ${ }^{4}$ Quan Liu, ${ }^{1}$ Rong Gui, ${ }^{1}$ \\ Juan Xiong, ${ }^{1}$ and Ming Yi $\mathbb{1}^{1}$ \\ ${ }^{1}$ Department of Physics, College of Science, Huazhong Agricultural University, Wuhan, China \\ ${ }^{2}$ Institute of Applied Physics, Huazhong Agricultural University, Wuhan, China \\ ${ }^{3}$ Department of Physics and Institute of Biophysics, Central China Normal University, Wuhan, China \\ ${ }^{4}$ Nonlinear Research Institute, Baoji University of Arts and Sciences, Baoji, China \\ Correspondence should be addressed to Ming Yi; 366212684@qq.com
}

Received 27 October 2017; Revised 12 January 2018; Accepted 24 January 2018; Published 20 February 2018

Academic Editor: Dimitri Volchenkov

Copyright (C) 2018 Yuangen Yao et al. This is an open access article distributed under the Creative Commons Attribution License, which permits unrestricted use, distribution, and reproduction in any medium, provided the original work is properly cited.

\begin{abstract}
Neurons can detect weak target signals from complex background signals through stochastic resonance (SR) and vibrational resonance (VR) mechanisms. However, random phase variation of rapidly fluctuating background signals is generally ignored in classical VR or SR studies. Here, the rapidly fluctuating background signals are modeled by bounded noise with random rapidly fluctuating phase derived from Wiener process. Then, the influences of bounded noise on the weak signal detection are discussed in the FitzHugh-Nagumo (FHN) neuron. Numerical results reveal the occurrence of bounded noise-induced single- and biresonance as well as a transition between them. Randomness in phase can enhance the adaptability of neurons, but at the cost of signal detection performance so that neurons can work in more complex environments with a wider frequency range. More interestingly, bounded noise with appropriate parameters can not only optimize information transmission but also simultaneously reduce energy consumption. Finally, the potential mechanism of bounded noise is explained.
\end{abstract}

\section{Introduction}

Neurons are the basic information processing devices of the nervous system, and they work in very noisy and complex environments $[1,2]$. The roles and environmental features of neurons demand them to reliably detect extremely weak extrinsic target stimuli (or signals) from noisy background signals with certain specialized mechanisms [1]. Therefore, a pivotal and interesting problem in neuroscience is to explore the relation between noise and signal detection and to look for signal detection mechanisms in the nervous system [2]. In the last century, many efforts had revealed the constructive role of noise. While investigating the periodically recurrent ice ages, Benzi and collaborators firstly discovered noiseinduced stochastic resonance (SR) where an external weak signal can be enhanced and optimized by noise [3]. Later, it was discovered that noise can also induce coherent oscillations in a simple two-dimensional system even without an external signal [4]. This noise-induced coherent oscillation phenomenon (called coherence resonance) also appears in the excitable FitzHugh-Nagumo (FHN) system subjected only to noise [5]. In fact, noise-induced resonance can be widely observed in various neuronal models, such as Hodgkin-Huxley (HH) [6], Hindermarsh-Rose (HR) [7], and Morris-Lecar (ML) [8]. In particular, in the last two decades, many efforts revealed that sensory systems can use noises to enhance their sensitivity for detecting weak periodic signals [9-11]. Therefore, noise-induced enhancement of response is considered to be a possible mechanism for signal detection.

Nonlinear systems are driven by two periodic forces: a low-frequency (LF) one (considered as a signal) and an HF one (considered as a carrier) [12]. The HF force with an appropriate strength and frequency may augment the processing of a weak LF signal [13]. This phenomenon is called vibrational resonance (VR) [14]. It should be mentioned 
that two-frequency driving systems can be found in many different fields, such as the communication field where HF carrier waves are usually used to enhance the target signal [13], neuroscience [15], acoustics [16], and laser physics [17]. The influences of VR on signal detection and amplification have been widely investigated in these systems subjected to LF and HF driving forces. For example, Ullner et al. studied the influence of additive noise and the interplay between SR and VR and found that the response of an excitable system to a low-frequency signal can be enhanced by high-frequency driving [12]. Deng et al. investigated the effect of HF force on the detection of a subthreshold low-frequency signal in neuron populations with different topology structures and hybrid synapses $[18,19]$. Yao and Zhan found that signal detection and transmission can be effectively enhanced by VR in oneway coupled bistable systems [20] or a spatially periodic force in the reaction-diffusion system [21]. In our previous study, the occurrence and mechanism of VR were discussed in detail within the whole parameter plane of amplitude and frequency of HF force based on an excitable FHN neuron model driven by two regular periodic signals [13]. We found that VR can be induced by the transition between different phase-locking modes since each maximum of response measure appears exactly at the transition boundary of phase patterns of the LFsignal-free FHN model [13]. Therefore, VR is also regarded as a possible mechanism for understanding how neurons extract target signals from a noisy environment. As a matter of fact, besides noise and HF force, there are many other factors that influence signal detection, such as network topology [22], autapse [23-25], time delay [26, 27], and electromagnetic induction [28-30].

However, most previous studies mainly paid much attention to individual or combined effects of amplitude and frequency of HF force and did not consider the effect of random phase variation on the response of excitability systems [13]. In fact, besides amplitude and frequency, phase is also an important factor that influences the response of excitability systems to external signals $[1,31,32]$. Furthermore, real-world external signals are always irregular and the phase of signals may vary randomly with time. For example, a time-varying phase occurs when a periodic wave travels through a random medium or interface [33].

It is very important to find a model that appropriately describes the effects of noise when investigating a dynamical system under random perturbation. Gaussian noise is usually adopted in many cases for convenience of analysis. It is worth mentioning that the widely used Gaussian noise has the probability of taking very large values, which violates the notion that real physical quantities in real systems always take values in bounded intervals [34-36]. In fact, unbounded Gaussian noise is not always suitable for modeling all realistic random processes. Many stochastic processes in real-life systems do not follow either white or colored Gaussian laws [37]. Moreover, most parameters in real-life systems are required to be strictly positive quantities, such as the time-varying fluctuation of reaction rates [37]. If unbounded Gaussian noise is used rather than bounded noise, unrealistic model-based inferences may occur due to the infinite domain of Gaussian noise [37]. For example, biologically paradoxical results occur in the pharmacokinetics model of antitumor chemotherapy: antitumor drugs have the probability of increasing the number of the tumor cells $[37,38]$. Moreover, experimental studies in sensory and other biological systems support the necessity of using nonGaussian noises [39-41]. More importantly, Gaussian noise is inappropriate to directly mimic the signals with a rapidly fluctuating phase. The rapidly fluctuating background signals around neurons can be modeled by bounded noise with a random rapidly fluctuating phase derived from the Wiener process. In addition, bounded noise is a simple non-Gaussian noise in mathematical presentation, and its spectrum may be either narrowband or broadband by appropriately choosing relevant parameters [42]. Therefore, as a reasonable and versatile random model, it has been long used in electrical engineering and has been recently used in mechanical and structural engineering and biological systems [43-45].

Many bounded noise-induced phenomena, such as resonance $[46,47]$, transitions $[39,48]$, and spatial synchronization [42], have been reported. We have also investigated the effect of bounded noise on the formation and instability of spiral waves $[35,36]$. In particular, the combined effects of correlated bounded noises and weak periodic signal were investigated in a recent study, and related results suggest that bounded noise can enhance the detection of external signals $[49,50]$. Although the effects of the time-varying initial phase of the received signal on signal detection have been reported in excitable systems using phase noise to mimic the timevarying initial phase of the target signal [1, 51-53], the related studies mainly focus on target signals and take no account of rapidly fluctuating background signals received by neurons. To the best of our knowledge, the resonance behavior and signal detection and amplification in rapidly fluctuating background signals have not previously been studied. In this study, the rapidly fluctuating background signals are modeled by bounded noise with constant amplitude and random rapidly fluctuating phase derived from the Wiener process. Then, the effects of bounded noise on signal detection and amplification are mainly investigated based on the Fourier coefficient $Q$ for measuring the system response to input signals.

\section{Model and Simulation}

As a simplified variant of the famous Hodgkin-Huxley model, the FHN model is simple but can represent the major characteristics of the electrophysiological activity of neurons. The FHN model is described by the following coupled equations $[13,15]$.

$$
\begin{aligned}
\varepsilon \frac{\mathrm{d} x}{\mathrm{~d} t} & =x-x^{3}-y+I+A \cos (\omega t)+\xi \\
\frac{\mathrm{d} y}{\mathrm{~d} t} & =4 x-y+2.8,
\end{aligned}
$$

where fast variable $x(t)$ stands for the membrane voltage of the neuron, whereas slow variable $y(t)$ is linked to the conductivity of the potassium channels embedded in the neuron membrane. A small parameter of time scale $\varepsilon=0.02$ results 
in the separation of all motions into fast and slow ones. The characteristics of solutions of the system are controlled by a time independent external signal $I$. Only one stable excitable steady state occurs and the system is excitable when $I$ is less than 0.898 . Small but finite deviations from steady state may give rise to a transition to the unstable point $x=y=1$ only after a large excursion. Here, $I$ is set to 0 throughout this paper. $A \cos (\omega t)$ denotes an external LF periodic signal. We set $A=0.32$ and $\omega=0.3$ throughout this paper so that this signal is too weak to evoke firing by itself. Namely, this signal is subthreshold.

In (1), $\xi$ represents the external bounded noise with a random rapidly fluctuating phase. For convenience, we call it simply HF bounded noise or irregular HF signal in the rest of this article. Notice that HF bounded noise is not an exclusive name for noise. The expression of $\xi$ is described as follows [44]:

$$
\xi=B \cos (N \omega t+\sigma W(t))=B \cos \left(\omega_{B} t+\sigma W(t)\right),
$$

where $B$ denotes the amplitude of the external HF bounded noise and the angular frequency $\omega_{B}=N \omega$ of HF bounded noise is $N(>1)$ times the angular frequency $\omega$ of the LF signal; $W(t)$ is the unit Wiener process, and $\sigma$ denotes the intensity of the unit Wiener process $W(t)$. For $t \rightarrow \infty$, the mean, autocorrelation function, and power spectral density of bounded noise $\xi$ are, respectively, represented as follows [44]:

$$
\begin{aligned}
& \langle\xi(t)\rangle=0, \\
& \langle\xi(t) \xi(t+\tau)\rangle=\frac{B^{2}}{2} \exp \left(-\frac{\sigma^{2} \tau}{2}\right) \cos \left(\omega_{B} \tau\right), \quad \tau>0, \\
& S\left(\omega_{B}^{\prime}\right) \\
& =\frac{(B \sigma)^{2}}{2 \pi}\left[\frac{1}{4\left(\omega_{B}-\omega_{B}^{\prime}\right)^{2}+\sigma^{4}}+\frac{1}{4\left(\omega_{B}+\omega_{B}^{\prime}\right)^{2}+\sigma^{4}}\right] .
\end{aligned}
$$

Clearly, (6) shows two symmetrical peaks at $\omega_{B}^{\prime}= \pm \omega_{B}$. When $\omega_{B} \rightarrow 0$, the two peaks merge into one. The position of the peak of power spectral density is mainly dominated by $\omega_{B}$, and the bandwidth of bounded noise mainly depends on the value of $\sigma$. Thus, the spectrum of bounded noise may be either narrowband or broadband by appropriately choosing relevant parameters. It turns into a narrowband process when $\sigma$ is small enough, whereas it approaches white noise when $\sigma \rightarrow$ $\infty$. The random phase fluctuation of bounded noise can be tuned by changing $\sigma$, and larger $\sigma$ results in faster fluctuation (Figure 1). When $\sigma$ is sufficiently large, the phase of $\xi$ is mainly dominated by Wiener process and the behavior of $\xi$ is completely like bounded noise rather than a regular HF periodic signal. Conversely, the decreasing $\sigma$ makes the behavior of $\xi$ more like a regular periodic signal. In particular, bounded noise turns into a cosine periodic signal when $\sigma$ is equal to 0 (Figure 1(a)). Therefore, in this study, the intensity $\sigma$ of the unit Wiener process, angular frequency ratio $N$, and amplitude of $\xi$ are important parameters that influence the weak signal detection. In the computation, bounded noise $\xi$ can be obtained by directly simulating the unit Wiener process [54]. As reported previously, the time evolution of a unit Wiener process is determined in our numerical simulations by the following formula $[35,48]$ :

$$
W(t)=W(t-\Delta t)+\sqrt{-2 \Delta t \ln \chi_{1}} \cos \left(2 \pi \chi_{2}\right) .
$$

Here, $\chi_{1}$ and $\chi_{2}$ are two independent random numbers which are uniformly distributed on the unit interval. We set a time step $\Delta t=0.001$ time units through this paper. In addition, according to Euler's method, (1) and (2) are discretized in our numerical simulations as follows:

$$
\begin{aligned}
x(t+ & \Delta t) \\
= & x(t) \\
& +\frac{1}{\varepsilon}\left[x(t)-x^{3}(t)-y(t)+I+A \cos (\omega t)\right] \Delta t \\
& +\frac{1}{\varepsilon} B \cos [N \omega t+\sigma W(t)] \Delta t, \\
y(t+ & \Delta t)=y(t)+[4 x(t)-y(t)+2.8] \Delta t .
\end{aligned}
$$

In this study, the Fourier coefficient (also called response measure and synchronization factor) $Q$ is used to quantitatively measure the correlation between the input frequency $\omega$ and the output temporal activity of the neuron. Its expression is as follows [13]:

$$
\begin{aligned}
Q & =\sqrt{Q_{\text {sin }}^{2}+Q_{\text {cos }}^{2}}, \\
Q_{\sin } & =\frac{\omega}{2 \pi m} \int_{T_{0}}^{T_{0}+2 \pi m / \omega} 2 x(t) \sin (\omega t) \mathrm{d} t, \\
Q_{\cos } & =\frac{\omega}{2 \pi m} \int_{T_{0}}^{T_{0}+2 \pi m / \omega} 2 x(t) \cos (\omega t) \mathrm{d} t .
\end{aligned}
$$

A sufficiently large $T_{0}=1,000$ time units is chosen to discard the transient process. A sufficiently large $m=500$ periods of weak LF periodic forcing is used in the calculation of Q. Large spikes rather than subthreshold oscillations carry information in neuronal systems [13]. Therefore, we set the threshold $x_{s}=0$ in the calculation of Q. If $x<x_{s}, x$ is replaced by a fixed value $x=-1$; otherwise, $x$ remains the same $[13,18]$. Larger $Q$ indicates better synchronization between input LF signal and the output of the system and thus also suggests more information transported through a particular forcing frequency $\omega[13]$.

\section{Main Results}

We firstly discuss how the intensity $\sigma$ of the unit Wiener process affects the output temporal activity of the neuron. For comparison, the LF input signals are also drawn and marked with red lines in Figure 2. When the amplitude and frequency of HF bounded noise are not too high, such as $B=0.2$ and $N=10^{0.56}$, for $\sigma=0$, the output of the system is below the threshold and cannot carry any information about LF signal (Figure 2(a)), and thus there is no synchronization between 


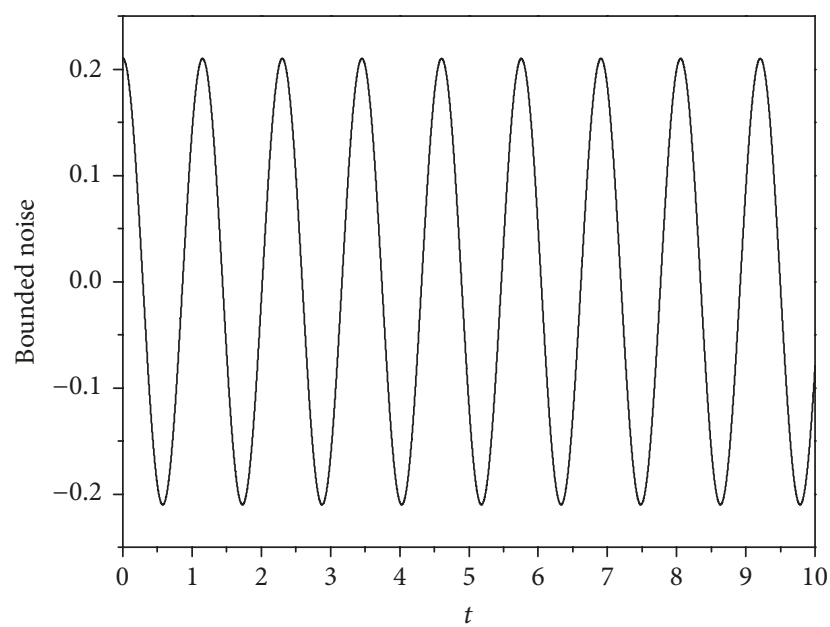

(a)

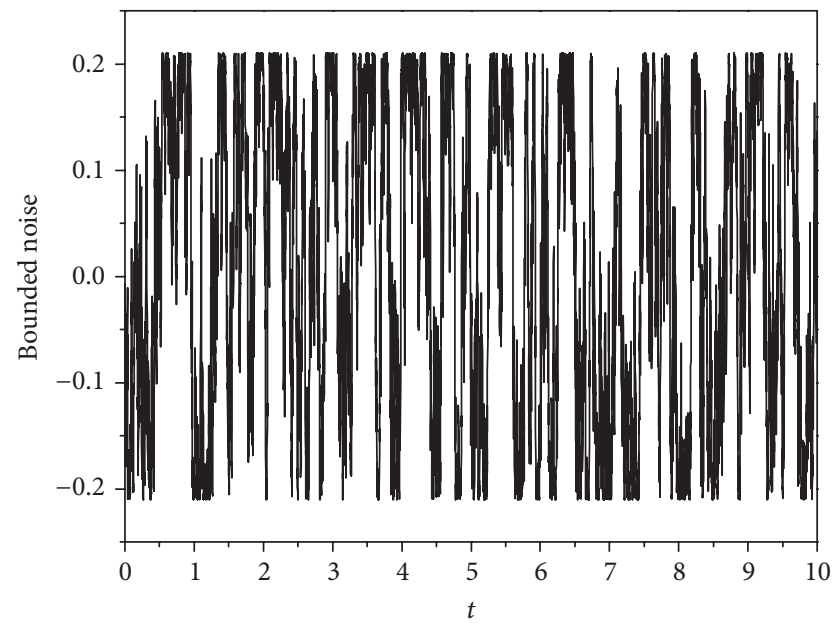

(c)

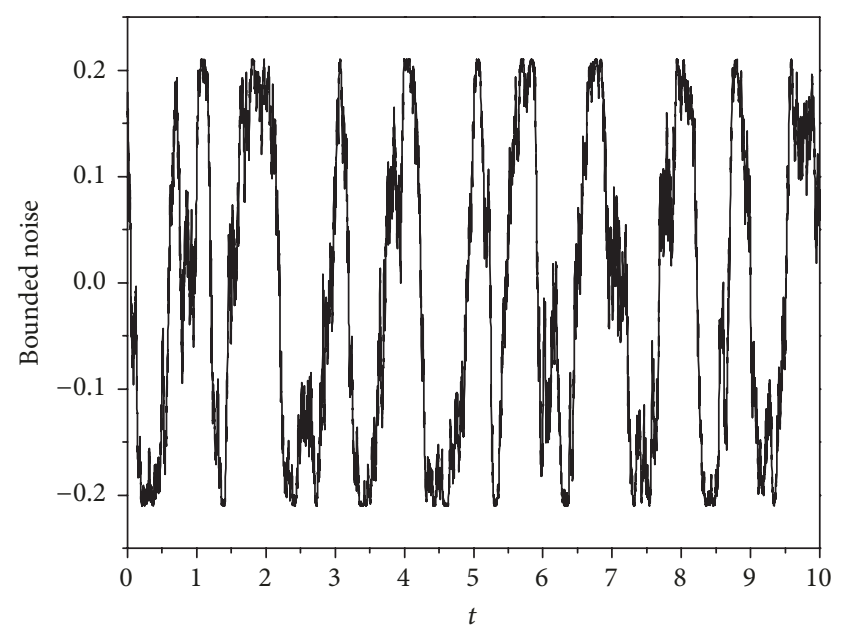

(b)

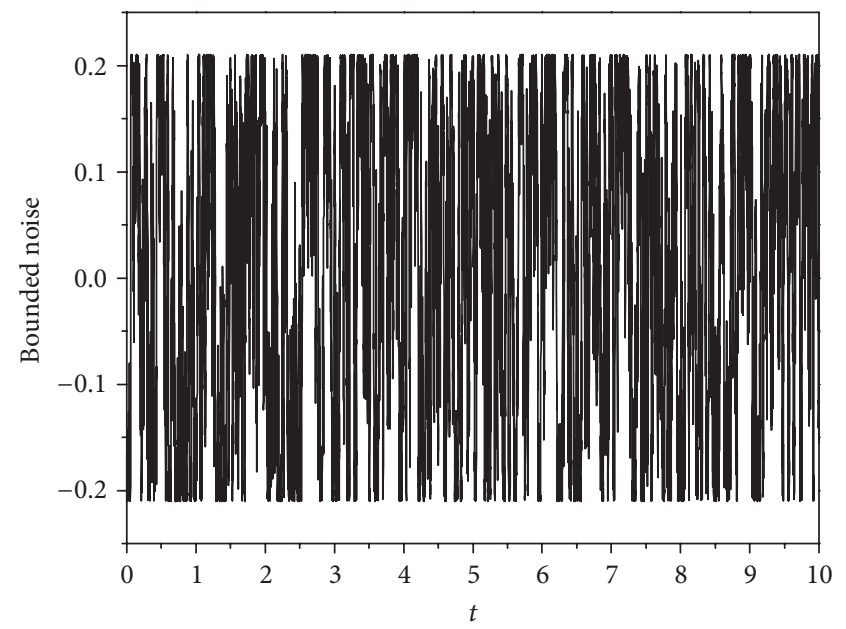

(d)

Figure 1: Time series of HF bounded noise $\xi=B \cos (N \omega t+\sigma W(t))$ for different intensities $\sigma \cdot B=0.2$ and $N=10^{0.56}$. (a) $\sigma=0$; (b) $\sigma=2$; (c) $\sigma=6$; (d) $\sigma=10$.

the input signal and the output of the system (Figure 2(a)). With the increase of $\sigma$, firing of system output begins to occur and the synchronization between the input signal and the output of the system gradually occurs during the positive half cycle of LF signal, while the response of the system fluctuates around the resting state during the negative half cycle of LF signal (Figures 2(b), 2(c), and 2(d)). The synchronization between the input signal and the output of the system is clearly observed and information carried by the LF input signal is amplified remarkably for an appropriate intensity $\sigma$ of the unit Wiener process (Figure 2(c)). However, if $\sigma$ is further increased, the synchronization between the input signal and the output of the system is decreased (Figure 2(d)), which implies that the capacity of information processing of neurons is degraded. Therefore, there is the optimal intensity $\sigma$ of the unit Wiener process to obtain the best performance of weak signal detection. In other words, HF bounded noiseinduced resonance can be observed.

To further confirm the above results quantitatively and investigate the effect of angular frequency ratio $N$, the curve of response measure $Q$ versus increasing $\log _{10} N$ is plotted in Figure 3 for different intensities $\sigma$ of the unit Wiener process. When the angular frequency ratio $N$ is small, for example, $\log _{10} N<1$, only a regular HF periodic signal (i.e., $\sigma=$ 0 ) is insufficient to excite firing of a neuron so that the value of $\mathrm{Q}$ is zero. However, with the increase of $\sigma, \mathrm{Q}$ increases firstly, reaches a maximum, and then decreases, which indicates that the existence of an optimal intensity $\sigma$ makes the LF input signal and the output of the system more synchronized (Figure 3(a)). When the angular frequency ratio $N$ is moderate, for example, $1<\log _{10} N<2$, the regular HF periodic signal itself (i.e., $\sigma=0$ ) is completely sufficient to excite firing of a neuron and the value of $Q$ in this case (i.e., $\sigma=0$ ) is larger than that in all other cases (i.e., $\sigma>0$ ) (Figure 3(a)), which suggests that HF bounded noise represses synchronization between the input signal and the output of the system. However, with the further increase of $\log _{10} N$, for example, $\log _{10} N>2$, HF bounded noise facilitates synchronization again (Figure 3(a)). Therefore, HF bounded noise can have a facilitation or repression effect on 


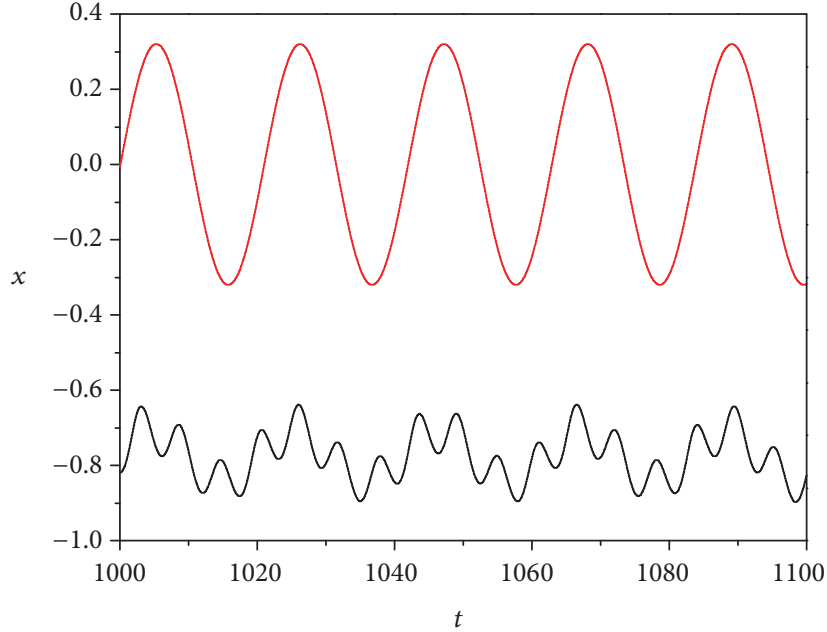

(a)

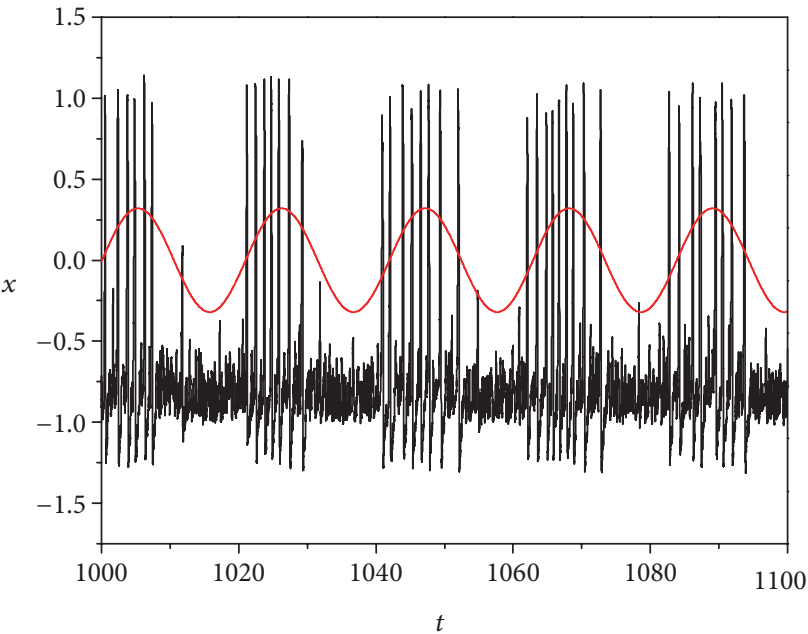

(c)

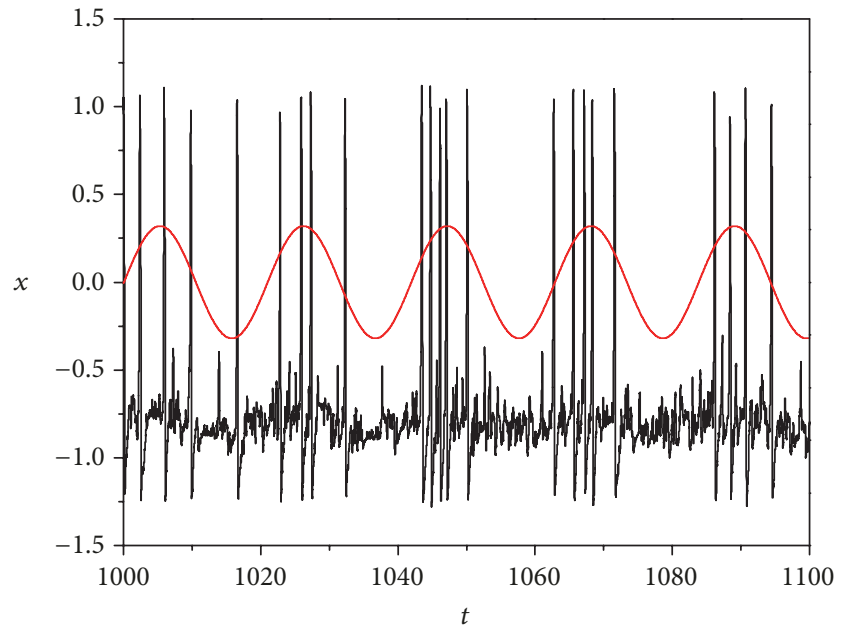

(b)

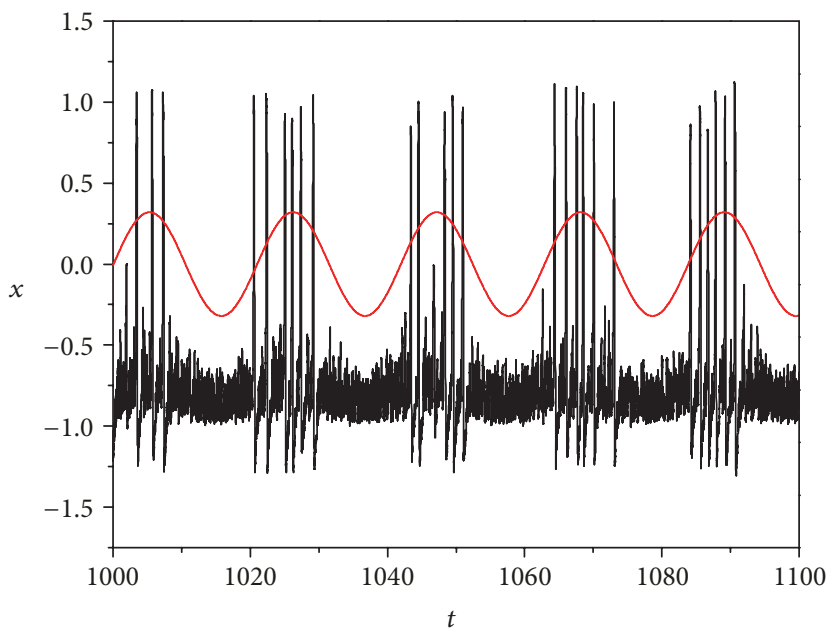

(d)

Figure 2: Time series of $x$ for different intensities $\sigma$. The LF signals are also drawn and marked with red lines for comparison. $B=0.2$ and $N=10^{0.56}$. (a) $\sigma=0$; (b) $\sigma=2$; (c) $\sigma=6$; (d) $\sigma=10$.

synchronization between the input signal and the output of the system. Furthermore, for small $\sigma$ (such as $\sigma=0$ and $\sigma=1$ ), two almost symmetrical peaks can be observed in the curve of $Q-\log _{10} N$, which indicates the existence of the biresonance phenomenon (Figure 3(a)). However, with the increase of $\sigma$, the two peaks turn into a single peak, which suggests a transition from biresonance to single resonance. With the further increase of $\sigma$, peaks finally disappear and a platform can be observed in a wider frequency range. The occurrence of a platform indicates that, for larger $\sigma$ (such as $\sigma=6$ and $\sigma=10$ ), the response measure $Q$ of the system is insensitive to the variation of the angular frequency ratio $N$. The values of $Q$ in a wider plateau region are not zero, but less than the optimal value of $Q$. This result suggests that randomness in phase (i.e., $\sigma>0$ ) is helpful in enhancing the adaptability of neurons, but at the cost of part of the performance, so that neurons can work in more complex environments with a wider frequency range. Moreover, to give a better global view of the above results, we further draw a landscape and contour plot of $Q$ with increasing $\sigma$ and $\log _{10} N$, as shown in Figure 3(b). Two peaks clearly indicate the biresonance phenomenon. The changes of $Q$ from two peaks to a single peak and even to a platform show resonance transitions and improvement of adaptability of neurons.

Next, we investigate how the amplitude $B$ of HF bounded noise affects the response measure $Q$ of a system for angular frequency ratio $N$ and different intensities $\sigma$. When the angular frequency ratio $N$ is small, such as $N=10^{0.56}$ (Figure 4(a)), for $\sigma=0, Q$ increases at first, reaches a maximum of 0.11 near $B=0.9$, and then decreases (Figure 4(a)), which indicates that the existence of an optimal amplitude $B$ makes the output of system and LF input signal more synchronized. For $\sigma=1$, there is no clear peak in the curve of Q-B (Figure 4(a)). With the further increase of $\sigma$, a maximum of 0.13 can be observed evidently near $B=$ 0.2 (Figure 4(a)). Compared with the case of $\sigma=0$, the positions of the peaks for the case of $\sigma>0$ show a clear shift toward the left (lower amplitude), but the values of $Q$ 


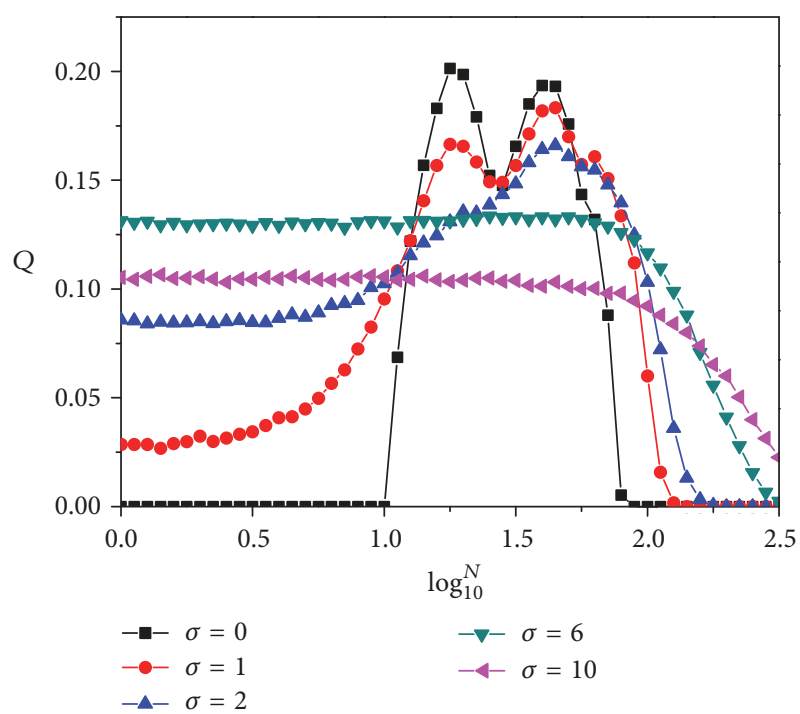

(a)

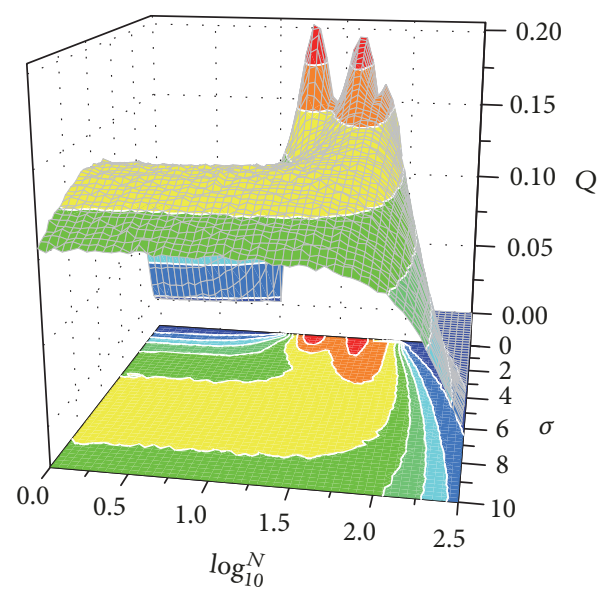

(b)

Figure 3: Dependencies of response measure $Q$ on increasing $\log _{10}{ }^{N}$ and $\sigma$ for $B=0.2$. The peaks indicate the occurrence of HF bounded noise-induced resonance phenomena. (a) $Q$ as a function of $\log _{10}{ }^{N}$ for different $\sigma$. (b) Landscape and contour plot of $Q$ with increasing $\log _{10}{ }^{N}$ and $\sigma$.

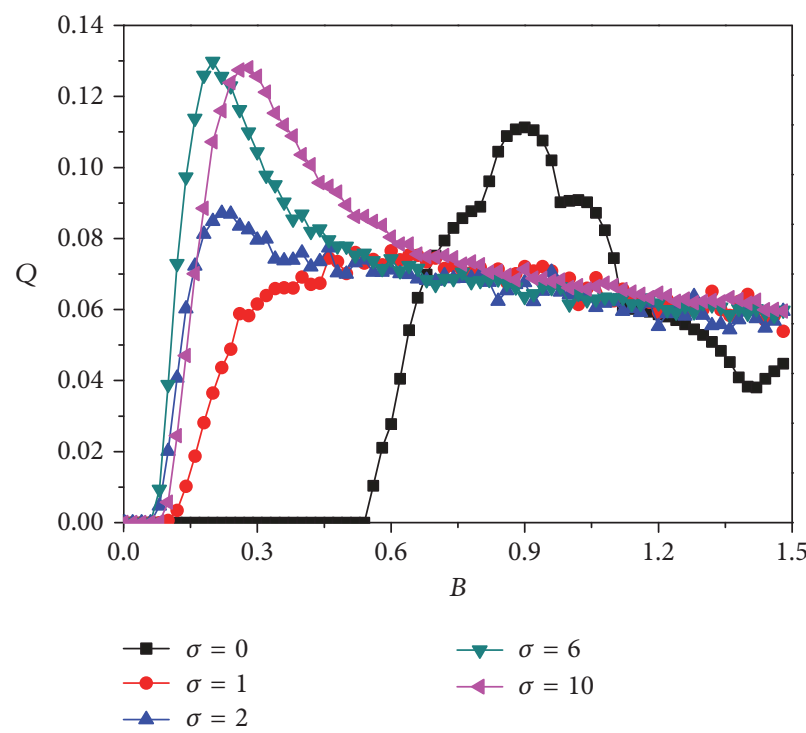

(a)

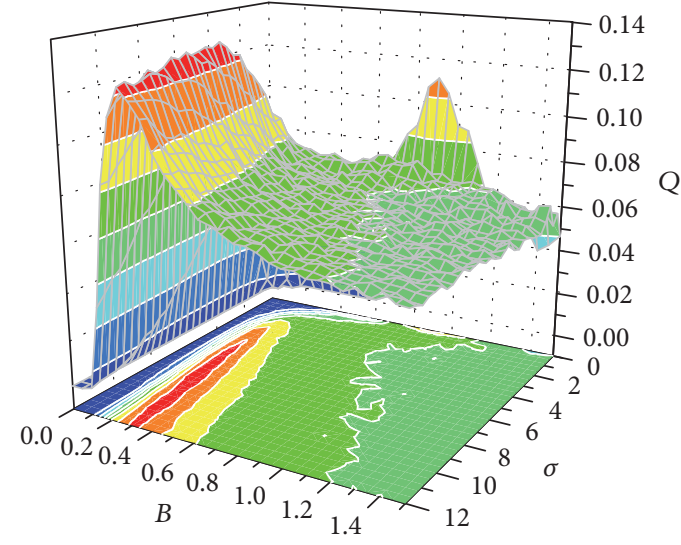

(b)

FIgURE 4: Dependencies of response measure $Q$ on increasing amplitude $B$ and intensity $\sigma$. The peaks indicate the occurrence of HF bounded noise-induced resonance phenomena. (a) $Q$ as a function of $B$ for different $\sigma$ when $N$ is set to $10^{0.56}$. (b) Landscape and contour plot of $Q$ with increasing $B$ and $\sigma$ when $N$ is set to $10^{0.56}$.

$(Q \approx 0.13)$ in the cases of $\sigma=6$ and $\sigma=10$ are slightly larger than that $(Q \approx 0.11)$ in the case of $\sigma=0$ (Figure $4(\mathrm{a})$ ). These findings can be clearly observed in the landscape and contour plot of $Q$ (Figure 4(b)). It is noted that, for simple harmonic oscillation, the lower amplitude $B$ stands for the lower energy consumption. Thus, the above results suggest that HF bounded noise with appropriate parameters can not only optimize information transmission but also simultaneously reduce energy consumption.
In what follows, we investigate how angular frequency ratio $N$ and amplitude $B$ affect the response measure $Q$ of a system with different intensities $\sigma$. To get a global view, the contour plots of $Q$ in the $B-\log _{10} N$ plane are depicted for different intensities $\sigma$ in Figure 5. There are some red regions, which show the occurrence of the resonance phenomenon (Figure 5(a)). Particularly, the weak LF input signal is enhanced greatly in the two deep red regions (Figure 5(a)). In addition, when $B$ is fixed, $Q$ varies with $\log _{10} N$, and the plots 


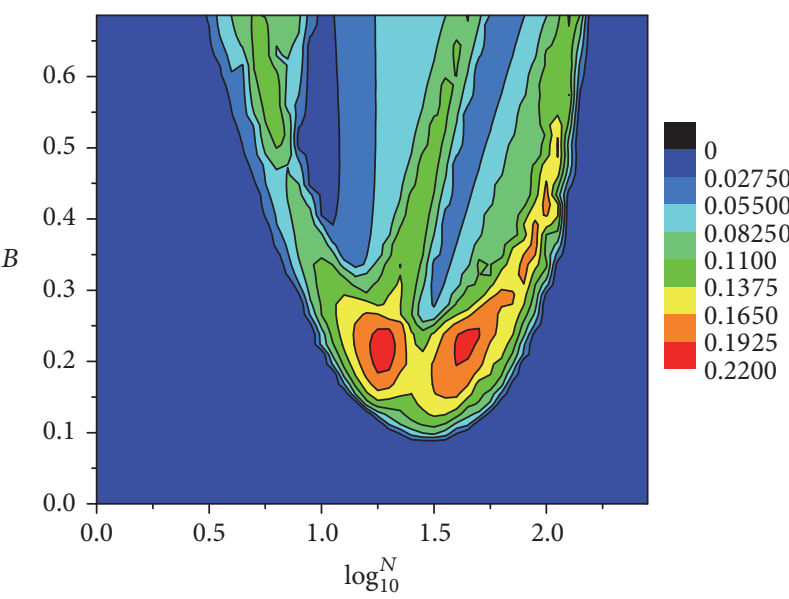

(a)

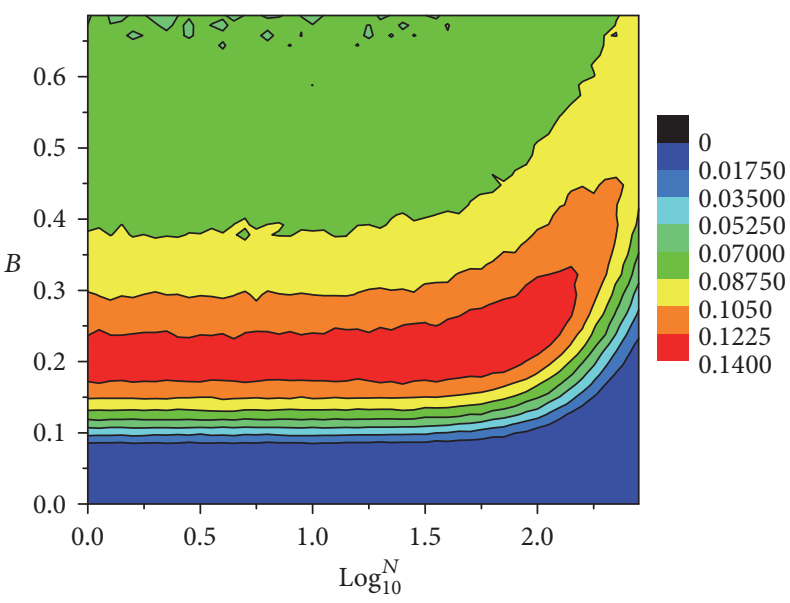

(b)

Figure 5: Contour plots of $Q$ in the $B-\log _{10}{ }^{N}$ plane for different intensities $\sigma$. (a) $\sigma=0$; (b) $\sigma=6$. Red indicates higher values of $Q$, as shown by the color bar.

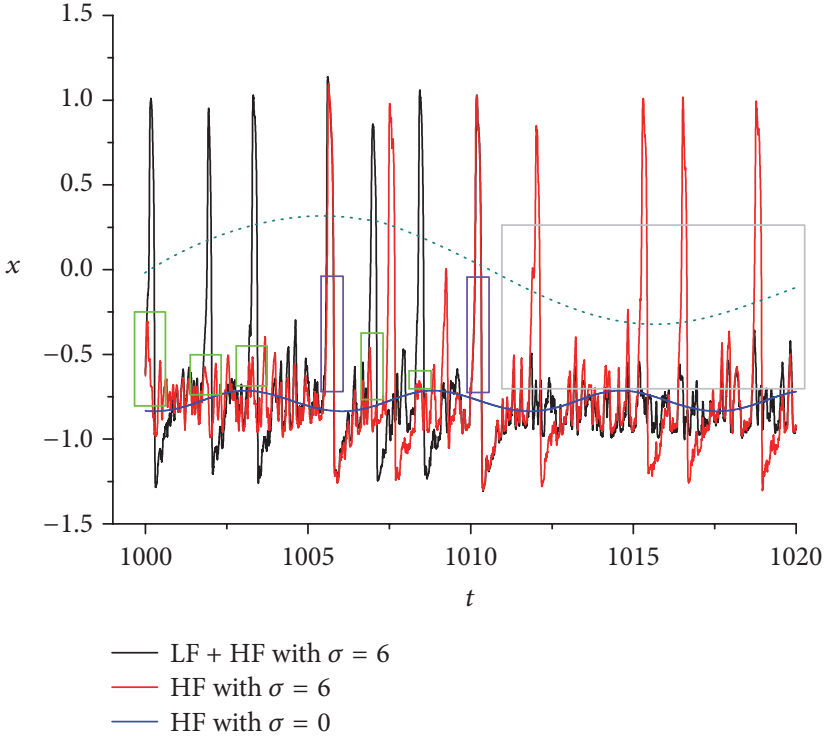

(a)

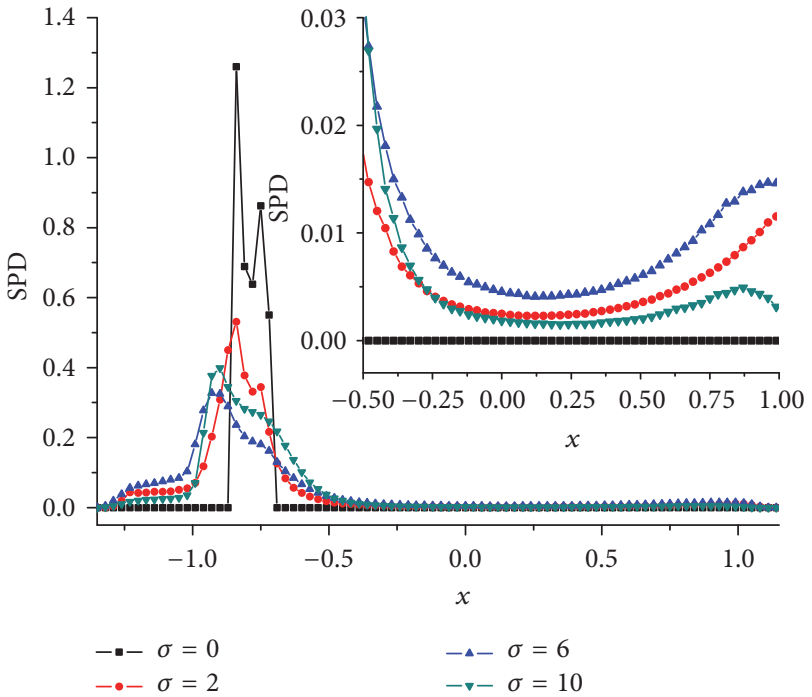

(b)

FIGURE 6: The potential mechanism analyses of HF bounded noise. $B=0.2$ and $N=10^{0.56}$. (a) Time series of $x$ of FHN neuron model separately or simultaneously subjected to LF signal, regular HF signal, and irregular HF signal during one cycle of LF signal denoted by the dotted line. (b) The steady-state probability distribution (SPD) as a function of $x$ for different $\sigma$.

of Q versus $\log _{10} N$ present a resonant behavior (Figure 5(a)). Moreover, the intensity $\sigma$ of the unit Wiener process of HF bounded noise can alter significantly the topography of contour plots (Figure 5). Overall, for larger $\sigma$, the optimal value of $Q$ is smaller, but the frequency range where $Q$ has suboptimal values is greatly expanded (Figure 5(b)), which suggests that neurons may balance between using randomness in phase to enhance the performance of signal detection and strengthening their capacity to adapt to the complex environment.

These phenomena raise a question: what is the mechanism of HF bounded noise? To answer this problem, we plot the time series of the FHN model during a period of LF signal in Figure 6(a), where the dotted line denotes LF input signal for clarity. During the whole period of LF signal, the neuron subjected to only regular HF periodic signal (i.e., $\sigma=0$ ) cannot fire (Figure 6(a)), whereas the neuron subjected to only irregular HF signal (i.e., $\sigma=6$ ) can fire during the positive and negative half cycles of LF signal (Figure 6(a)). Therefore, we assume that randomness in phase makes neurons more easily go into a firing or excitable state even without LF input signal. In order to more clearly illustrate this point, the regular LF signal and irregular HF signal (i.e., $\sigma=6$ ) are simultaneously imposed on a neuron; we can observe that 
the neuron can fire during the positive half cycle of LF signal (Figure 6(a)). From the green boxes in Figure 6(a), we can see that the values of $x$ of the neuron subjected to the regular HF period signal (i.e., $\sigma=0$, blue line) are lower than that of the neuron subjected to an irregular HF period signal (i.e., $\sigma=$ 6 , red line). Considering that, the green boxes denote where neurons subjected to LF signal and irregular HF signal (i.e., $\sigma$ $=6$, black line) fire (Figure 6(a)). Consequently, we can come to a conclusion that the neuron subjected to the irregular HF signal (i.e., $\sigma=6$ ) is virtually in an excitable state (marked by green boxes) or firing state (marked by violet boxes), and the neuron can fire once there is an LF input signal and the neuron is in the positive half cycle of LF signal. In addition, we also observe that neurons subjected to LF signal and irregular HF signal (i.e., $\sigma=6$ ) cannot fire during the negative half cycle of LF signal (marked by a gray box) (Figure 6(a)). Compared with that, neurons subjected to only irregular HF signals (i.e., $\sigma=6$ ) can fire during this time; the above observations can be explained by the fact that the values of LF signal are less than zero during the negative half cycle of LF signal so that the LF signal suppresses the firing of the neuron. Furthermore, the steady-state probability distribution (SPD) of $x$ is used to discuss the mechanism of HF bounded noise. Figure 6(b) shows that the unimodal SPD near -0.85 of $x$ turns into a bimodal structure that has the second peak near 1 (as shown in the inset of Figure 6(b)). As shown in Figure 6, with the increase of $\sigma$, nonzero values of $x$ appear between -0.5 and 1, which means HF bounded noise-induced transition from resting state to excitable state or even to firing state (Figure 6(b)). Taken together, the potential mechanism of HF bounded noise is that randomness in phase makes neurons more easily go into a firing or excitable state.

\section{Conclusions}

Real neurons are often surrounded in very noisy environments. Neurons are requested to detect generally feeble target signals (or stimuli) from noisy background signals. It is very interesting and significant to elucidate the mechanisms of weak signal detection in an excitable nervous system. A number of efforts have been contributed to this area. But the understanding of these mechanisms for a nervous system subjected to HF bounded noise has remained incomplete. Bounded noise with constant amplitude and time-varying random phase not only is a versatile model for simulating irregularity included in real-world external signals, but also meets the fact that the real physical quantities in a real system are always bounded.

In this study, we study in detail the effect of HF bounded noise on the detection of a weak LF periodic signal in the FHN neuron. It is found that HF bounded noise has a facilitation or repression effect on synchronization between the input signal and the output of the system. Moreover, the numerical results also demonstrate the occurrence of $\mathrm{HF}$ bounded noise-induced single- and biresonance as well as a transition between them. Through this resonance mechanism, the response of neurons in random rapidly fluctuating background signals to the external subthreshold target signal can be significantly enhanced. The numerical results also reveal that randomness in phase (i.e., $\sigma>0$ ) is helpful in enhancing the adaptability of neurons, but at the cost of part of the performance so that neurons can work in more complex environments with a wider frequency range. Therefore, neurons may balance between using randomness in phase to enhance the performance of signal detection and strengthening their capacity to adapt to complex environments. More interestingly, HF bounded noise with appropriate parameters can not only optimize information transmission, but also simultaneously reduce energy consumption. The above results may support the fact that the animals have the capacity to detect target signals from heterogeneous and complex signals and thus adapt to complex and fluctuating environments. We also attempt to qualitatively explain the potential mechanism of time-varying random phase through analyzing SPD and time series of $x$ of FHN neuron model separately or simultaneously subjected to LF signal, regular HF signal (i.e., $\sigma=0$ ), and irregular HF signal (i.e., $\sigma \neq 0$ ). The potential mechanism of HF bounded noise is that randomness in phase makes neuron more easily go into a firing or excitable state. All in all, the results we report here not only provide a possible mechanism for amplifying or detecting the weak signal in many fields, such as neuroscience, acoustics, and communication technologies, but also lay the foundations for related researches involving bounded noise.

\section{Conflicts of Interest}

The authors declare that they have no conflicts of interest.

\section{Authors' Contributions}

Yuangen Yao implemented the project, analyzed data, and wrote the manuscript. Lijian Yang, Canjun Wang, Quan Liu, Rong Gui, Juan Xiong, and Ming Yi extensively shared and discussed results. All authors read and approved the final manuscript.

\section{Acknowledgments}

This work was supported by the National Natural Science Foundation of China (Grants nos. 31601071, 11675060, 91730301, and 11205006), the Huazhong Agricultural University Scientific and Technological Self-Innovation Foundation Program (Grant no. 2015RC021), and the Natural Science New Star of Science and Technologies Research Plan in Shaanxi Province of China (Grant no. 2014KJXX-77).

\section{References}

[1] X. Liang, L. Zhao, and Z. Liu, "Phase-noise-induced resonance in a single neuronal system," Physical Review E: Statistical, Nonlinear, and Soft Matter Physics, vol. 84, no. 3, Article ID 031916, 2011.

[2] T. P. Vogels and L. F. Abbott, "Signal propagation and logic gating in networks of integrate-and-fire neurons," The Journal of Neuroscience, vol. 25, no. 46, pp. 10786-10795, 2005. 
[3] R. Benzi, A. Sutera, and A. Vulpiani, "The mechanism of stochastic resonance," Journal of Physics A: Mathematical and Theoretical, vol. 14, no. 11, pp. L453-L457, 1981.

[4] H. Gang, T. Ditzinger, C. Z. Ning, and H. Haken, "Stochastic resonance without external periodic force," Physical Review Letters, vol. 71, no. 6, pp. 807-810, 1993.

[5] A. S. Pikovsky and J. Kurths, "Coherence resonance in a noisedriven excitable system," Physical Review Letters, vol. 78, no. 5, pp. 775-778, 1997.

[6] S.-G. Lee, A. Neiman, and S. Kim, "Coherence resonance in a Hodgkin-Huxley neuron," Physical Review E: Statistical Physics, Plasmas, Fluids, and Related Interdisciplinary Topics, vol. 57, no. 3, pp. 3292-3297, 1998.

[7] K. Wiesenfeld, D. Pierson, E. Pantazelou, C. Dames, and F. Moss, "Stochastic resonance on a circle," Physical Review Letters, vol. 72, no. 14, pp. 2125-2129, 1994.

[8] N. Montejo, M. N. Lorenzo, V. Pérez-Villar, and V. Pérez$\mathrm{Mu}$ ?Uzuri, "Noise correlation length effects on a Morris-Lecar neural network," Physical Review E Statistical Nonlinear \& Soft Matter Physics, vol. 72, Article ID 011902, 2005.

[9] J. K. Douglass, L. Wilkens, E. Pantazelou, and F. Moss, "Noise enhancement of information transfer in crayfish mechanoreceptors by stochastic resonance," Nature, vol. 365, no. 6444, pp. 337-340, 1993.

[10] K. Wiesenfeld and F. Moss, "Stochastic resonance and the benefits of noise: from ice ages to crayfish and SQUIDs," Nature, vol. 373, no. 6509, pp. 33-36, 1995.

[11] B. J. Gluckman, T. I. Netoff, E. J. Neel, W. L. Ditto, M. L. Spano, and S. J. Schiff, "Stochastic resonance in a neuronal network from mammalian brain," Physical Review Letters, vol. 77, no. 19, pp. 4098-4101, 1996.

[12] E. Ullner, A. Zaikin, J. García-Ojalvo, R. Báscones, and J. Kurths, "Vibrational resonance and vibrational propagation in excitable systems," Physics Letters A, vol. 312, no. 5-6, pp. 348-354, 2003.

[13] L. Yang, W. Liu, M. Yi et al., "Vibrational resonance induced by transition of phase-locking modes in excitable systems," Physical Review E: Statistical, Nonlinear, and Soft Matter Physics, vol. 86, no. 1, Article ID 016209, 2012.

[14] P. S. Landa and P. V. McClintock, "Vibrational resonance," Journal of Physics A: Mathematical and General, vol. 33, no. 45, pp. L433-L438, 2000.

[15] D. Cubero, J. P. Baltanás, and J. Casado-Pascual, "Highfrequency effects in the FitzHugh-Nagumo neuron model," Physical Review E: Statistical, Nonlinear, and Soft Matter Physics, vol. 73, no. 6, Article ID 061102, 2006.

[16] J. D. Victor and M. M. Conte, "Two-frequency analysis of interactions elicited by Vernier stimuli," Visual Neuroscience, vol. 17, no. 6, pp. 959-973, 2000.

[17] D.-C. Su, M.-H. Chiu, and C.-D. Chen, "Simple two-frequency laser," Precision Engineering, vol. 18, no. 2-3, pp. 161-163, 1996.

[18] B. Deng, J. Wang, X. Wei, K. M. Tsang, and W. L. Chan, "Vibrational resonance in neuron populations," Chaos: An Interdisciplinary Journal of Nonlinear Science, vol. 20, no. 1, Article ID 018001CHA, 2010.

[19] J. Sun, B. Deng, C. Liu et al., "Vibrational resonance in neuron populations with hybrid synapses," Applied Mathematical Modelling, vol. 37, no. 9, pp. 6311-6324, 2013.

[20] C. Yao and M. Zhan, "Signal transmission by vibrational resonance in one-way coupled bistable systems," Physical Review E Statistical Nonlinear \& Soft Matter Physics, vol. 81, Article ID 061129, 2010.
[21] C. Yao, Z. He, J. Luo, and J. Shuai, "Resonance induced by a spatially periodic force in the reaction-diffusion system," Physical Review E Statistical Nonlinear \& Soft Matter Physics, vol. 91, Article ID 052901, 2015.

[22] Z. Liu and T. Munakata, "Scale-free topology-induced double resonance in networked two-state systems," Physical Review E Statistical Nonlinear \& Soft Matter Physics, vol. 78, Article ID 046111, 2008.

[23] J. Ma, X. Song, J. Tang, and C. Wang, "Wave emitting and propagation induced by autapse in a forward feedback neuronal network," Neurocomputing, vol. 167, pp. 378-389, 2015.

[24] C. Wang, S. Guo, and Y. Xu, "Formation of autapse connected to neuron and its biological function," Complexity, vol. 2017, Article ID 5436737, 9 pages, 2017.

[25] J. Ma and J. Tang, "A review for dynamics in neuron and neuronal network," Nonlinear Dynamics, vol. 89, no. 3, pp. 15691578, 2017.

[26] C. Yao, J. Ma, C. Li, and Z. He, "The effect of process delay on dynamical behaviors in a self-feedback nonlinear oscillator," Communications in Nonlinear Science and Numerical Simulation, vol. 39, pp. 99-107, 2016.

[27] L.-F. Wang, K. Qiu, and Y. Jia, "Effects of time delays in a mathematical bone model," Chinese Physics B, vol. 26, no. 3, Article ID 030503, 2017.

[28] L. Lu, Y. Jia, W. Liu, and L. Yang, "Mixed stimulus-induced mode selection in neural activity driven by high and low frequency current under electromagnetic radiation," Complexity, vol. 2017, pp. 1-11, 2017.

[29] M. Ge, Y. Jia, Y. Xu, and L. Yang, "Mode transition in electrical activities of neuron driven by high and low frequency stimulus in the presence of electromagnetic induction and radiation," Nonlinear Dynamics, vol. 91, no. 1, pp. 515-523, 2018.

[30] C. Wang and J. Ma, "A review and guidance for pattern selection in spatiotemporal system," International Journal of Modern Physics B, Article ID 1830003, 2017.

[31] Y. Jia and H. Gu, "Transition from double coherence resonances to single coherence resonance in a neuronal network with phase noise," Chaos: An Interdisciplinary Journal of Nonlinear Science, vol. 25, no. 12, Article ID 123124, 2015.

[32] Y. Jia and H. Gu, "Phase noise-induced double coherence resonances in a neuronal model," International Journal of Modern Physics B, vol. 29, no. 20, Article ID 1550142, 2015.

[33] E. I. Volkov, E. Ullner, A. A. Zaikin, and J. Kurths, "Oscillatory amplification of stochastic resonance in excitable systems," Physical Review E: Statistical, Nonlinear, and Soft Matter Physics, vol. 68, no. 2, Article ID 026214, p. 026214/7, 2003.

[34] G. Q. Cai and C. Wu, "Modeling of bounded stochastic processes," Probabilistic Engineering Mechanics, vol. 19, no. 3, pp. 197-203, 2004.

[35] Y. Yao, H. Deng, M. Yi, and J. Ma, "Impact of bounded noise on the formation and instability of spiral wave in a 2D Lattice of neurons," Scientific Reports, vol. 7, Article ID 43151, 2017.

[36] Y. Yao, H. Deng, C. Ma, M. Yi, and J. Ma, "Impact of bounded noise and rewiring on the formation and instability of spiral waves in a small-world network of hodgkin-huxley neurons," PLoS ONE, vol. 12, no. 1, Article ID e0171273, 2017.

[37] S. De Franciscis, G. Caravagna, and A. D’Onofrio, “Bounded noises as a natural tool to model extrinsic fluctuations in biomolecular networks," Natural Computing, vol. 13, no. 3, pp. 297-307, 2014. 
[38] A. d'Onofrio, “"Fuzzy oncology”: Fuzzy noise induced bifurcations and their application to anti-tumor chemotherapy," Applied Mathematics Letters, vol. 21, no. 7, pp. 662-668, 2008.

[39] R. V. Bobryk and A. Chrzeszczyk, "Transitions induced by bounded noise," Physica A: Statistical Mechanics and its Applications, vol. 358, no. 2-4, pp. 263-272, 2005.

[40] S. M. Bezrukov and I. Vodyanoy, "Noise-induced enhancement of signal transduction across voltage-dependent ion channels," Nature, vol. 378, no. 6555, pp. 362-364, 1995.

[41] H. S. Wio and R. Toral, "Effect of non-Gaussian noise sources in a noise-induced transition," Physica D: Nonlinear Phenomena, vol. 193, no. 1-4, pp. 161-168, 2004.

[42] X. L. Yang, Y. B. Jia, and L. Zhang, "Impact of bounded noise and shortcuts on the spatiotemporal dynamics of neuronal networks," Physica A: Statistical Mechanics and its Applications, vol. 393, pp. 617-623, 2014.

[43] A. D'Onofrio and A. Gandolfi, "Resistance to antitumor chemotherapy due to bounded-noise-induced transitions," Physical Review E: Statistical, Nonlinear, and Soft Matter Physics, vol. 82, no. 6, Article ID 061901, 2010.

[44] D. Li, W. Xu, X. Yue, and Y. Lei, "Bounded noise enhanced stability and resonant activation," Nonlinear Dynamics, vol. 70, no. 3, pp. 2237-2245, 2012.

[45] A. d'Onofrio, Ed., Bounded noises in physics, biology, and engineering, Modeling and Simulation in Science, Engineering and Technology, Birkhäuser Springer, New York, NY, USA, 2013.

[46] Y. Chai, C. Wu, and D. Li, "Coherence resonance in the FitzHugh-Nagumo neurons models driven by bounded noise," Modern Physics Letters B, vol. 29, no. 22, Article ID 1550121, 2015.

[47] Y. Yao, M. Yi, and D. Hou, "Coherence resonance induced by cross-correlated sine-Wiener noises in the FitzHugh-Nagumo neurons," International Journal of Modern Physics B, 2017.

[48] W. Guo, L.-C. Du, and D.-C. Mei, "Transitions induced by time delays and cross-correlated sine-Wiener noises in a tumorimmune system interplay," Physica A: Statistical Mechanics and its Applications, vol. 391, no. 4, pp. 1270-1280, 2012.

[49] D. Tatchim Bemmo, M. Siewe Siewe, and C. Tchawoua, "Combined effects of correlated bounded noises and weak periodic signal input in the modified FitzHugh-Nagumo neural model," Communications in Nonlinear Science and Numerical Simulation, vol. 18, no. 5, pp. 1275-1287, 2013.

[50] Y. Yao, C. Ma, C. Wang, M. Yi, and R. Gui, "Detection of sub-threshold periodic signal by multiplicative and additive cross-correlated sine-Wiener noises in the FitzHugh-Nagumo neuron," Physica A Statistical Mechanics \& Its Applications, 2017.

[51] F. Chapeau-Blondeau, "Stochastic resonance at phase noise in signal transmission," Physical Review E: Statistical Physics, Plasmas, Fluids, and Related Interdisciplinary Topics, vol. 61, no. 1, pp. 940-943, 2000.

[52] X.-S. Kang, X.-M. Liang, and H.-P. Lü, "Enhanced response to subthreshold signals by phase noise in a hodgkin - Huxley neuron," Chinese Physics Letters, vol. 30, no. 1, Article ID 018701, 2013.

[53] X. Liang and Z. Liu, "Effect of initial phase diversity on signal detection in excitable systems," Science China Technological Sciences, vol. 59, no. 3, pp. 376-386, 2016.

[54] D. J. Higham, "An algorithmic introduction to numerical simulation of stochastic differential equations," SIAM Review, vol. 43, no. 3, pp. 525-546, 2001. 


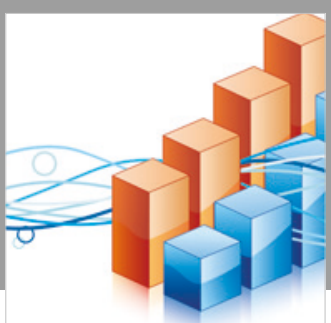

Advances in

Operations Research

\section{-n-m}
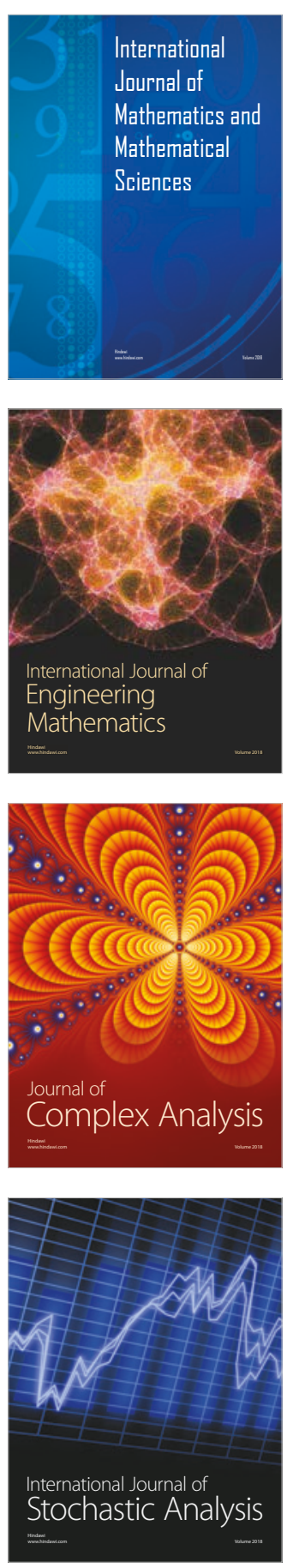
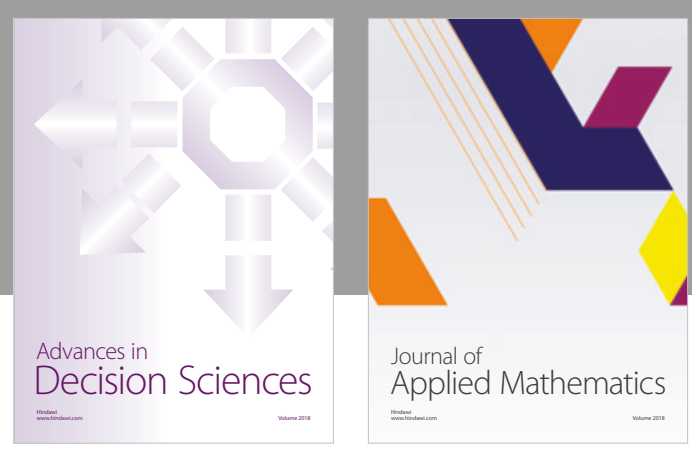

Journal of

Applied Mathematics
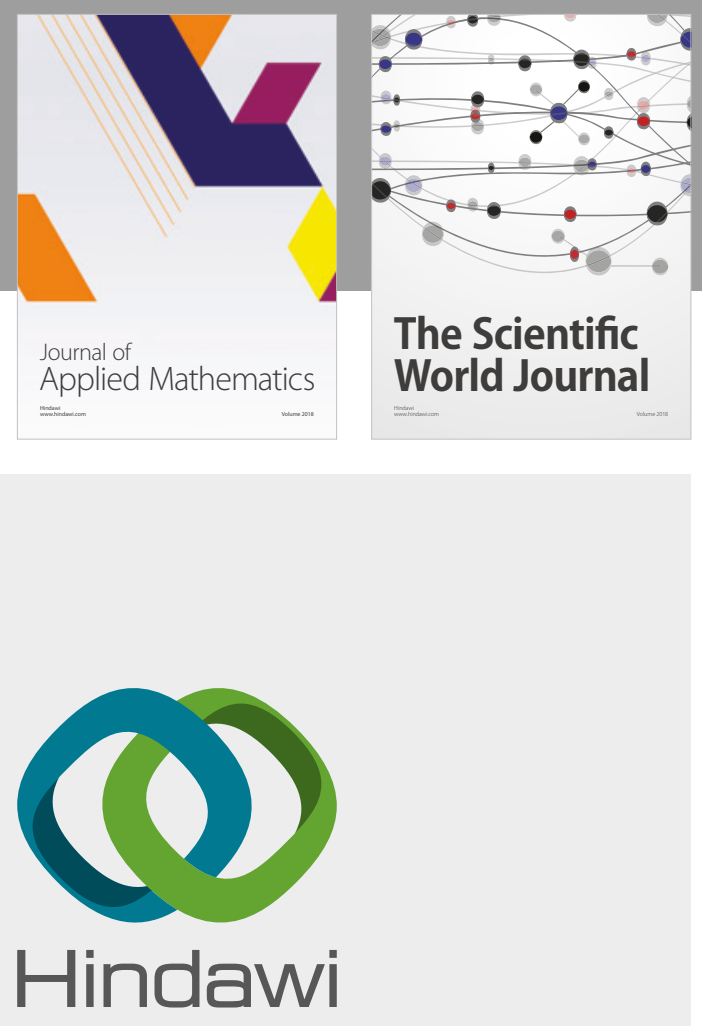

Submit your manuscripts at

www.hindawi.com

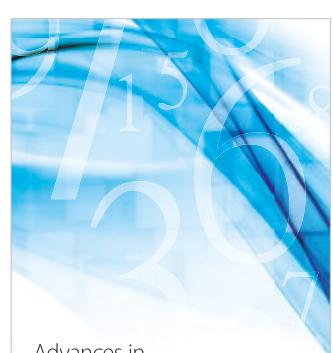

Advances in
Numerical Analysis
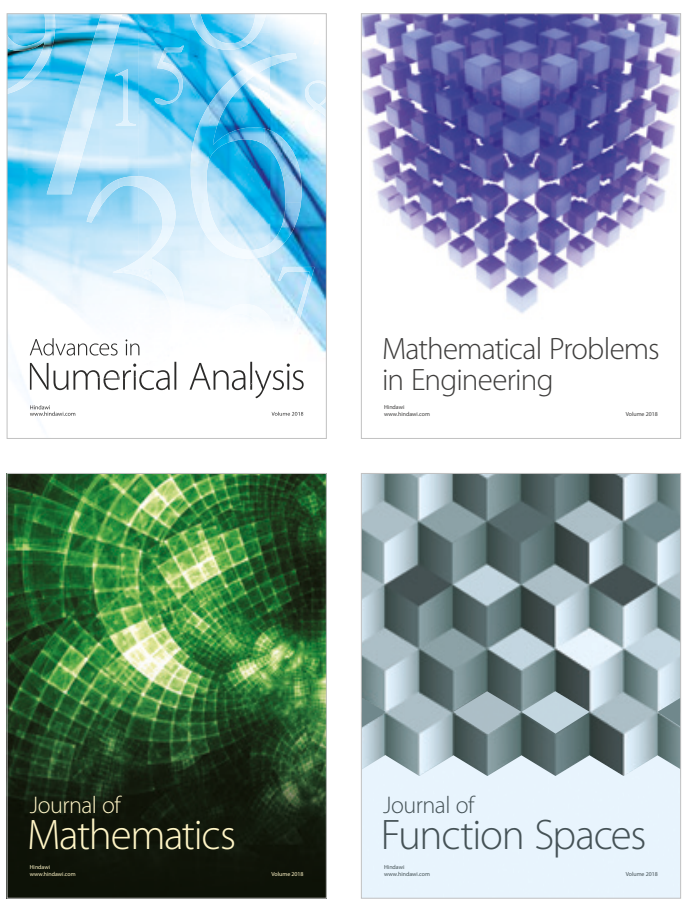

Mathematical Problems in Engineering

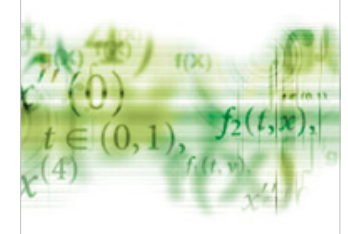

International Journal of

Differential Equations

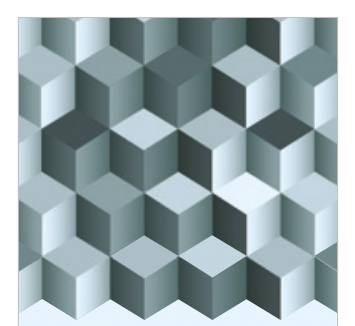

Journal of

Function Spaces
The Scientific

World Journal

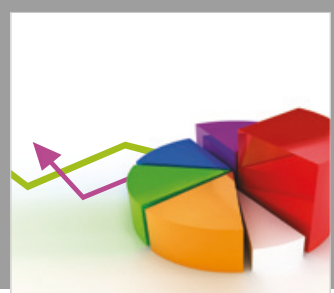

Journal of

Probability and Statistics
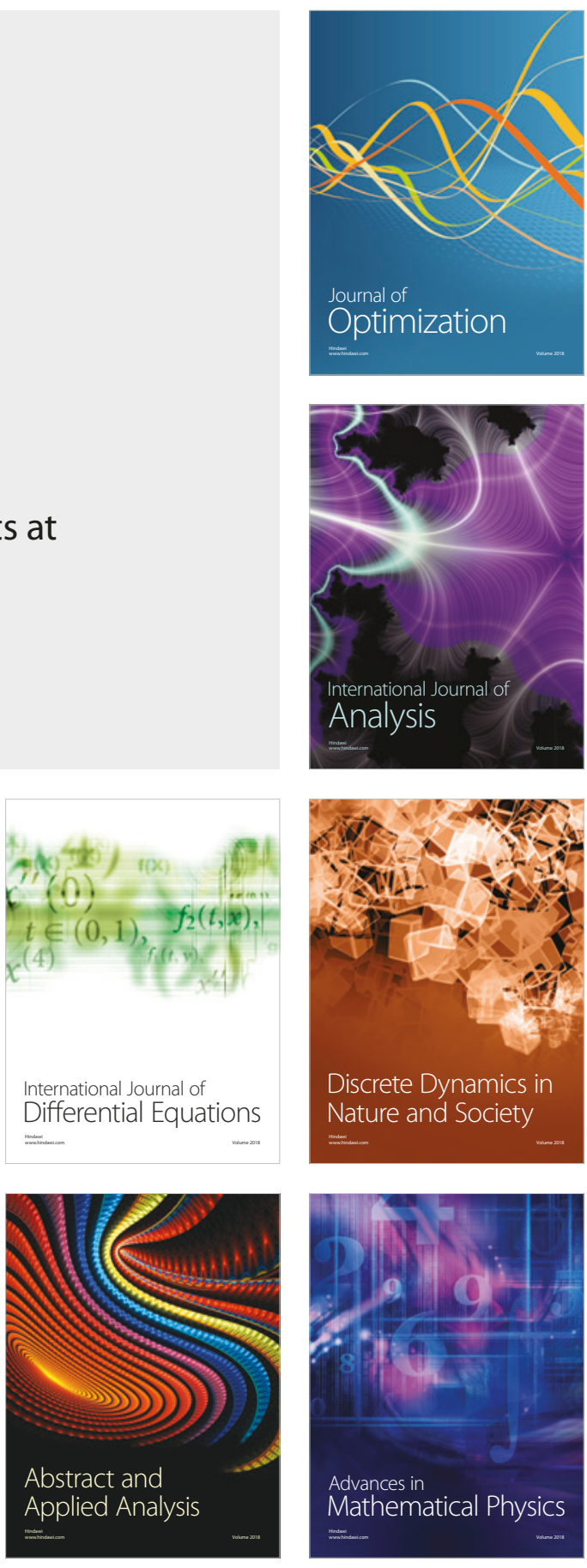